\title{
Polarization of x-gamma radiation produced by a Thomson and Compton inverse scattering
}

\author{
V. Petrillo, ${ }^{1,2}$ A. Bacci, ${ }^{2}$ C. Curatolo,,${ }^{1,2}$ I. Drebot,${ }^{2}$ A. Giribono, ${ }^{4}$ C. Maroli ${ }^{1}$ A. R. Rossi, ${ }^{2}$ \\ L. Serafini, ${ }^{2}$ P. Tomassini, ${ }^{1}$ C. Vaccarezza, ${ }^{3}$ and A. Variola ${ }^{3}$ \\ ${ }^{1}$ Università degli Studi di Milano, via Celoria 16, 20133 Milano, Italy \\ ${ }^{2}$ INFN-Sezione di Milano, via Celoria 16, 20133 Milano, Italy \\ ${ }^{3}$ INFN Laboratori Nazionali di Frascati,Via E. Fermi 44, 00044 Frascati, Roma, Italy \\ ${ }^{4}$ Università La Sapienza, Via Antonio Scarpa, 1400161 Roma, Italy \\ and INFN-Romal, Piazzale Aldo Moro, 200161 Rome, Italy
}

(Received 24 June 2015; published 19 November 2015)

\begin{abstract}
A systematic study of the polarization of $x$-gamma rays produced in Thomson and Compton scattering is presented, in both classical and quantum schemes. Numerical results and analytical considerations let us to establish the polarization level as a function of acceptance, bandwidth and energy. Few sources have been considered: the SPARC_LAB Thomson device, as an example of a x-ray Thomson source, ELI-NP, operating in the gamma range. Then, the typical parameters of a beam produced by a plasma accelerator has been analyzed. In the first case, with bandwidths up to $10 \%$, a contained reduction $(<10 \%)$ in the average polarization occurs. In the last case, for the nominal ELI-NP relative bandwidth of $5 \times 10^{-3}$, the polarization is always close to 1 . For applications requiring larger bandwidth, however, a degradation of the polarization up to $30 \%$ must be taken into account. In addition, an all optical gamma source based on a plasma accelerated electron beam cannot guarantee narrow bandwidth and high polarization operational conditions required in nuclear photonics experiments.
\end{abstract}

DOI: 10.1103/PhysRevSTAB.18.110701

PACS numbers: $41.50 .+\mathrm{h}$

\section{INTRODUCTION}

The development of $\mathrm{x}$ and gamma-ray sources with large spectral flux and high tunability opens the way to applications at the frontier of science [1,2], allowing us to deepen the fundamental knowledge and understanding of the properties of materials and living systems by probing the matter on microscopic-to-nuclear scales in space and time [2]. Thomson [3-10] and Compton sources [11,12] are among the most performing devices in producing radiation with short wavelength, high power, ultrashort time duration, large transverse coherence and tunability. In the existing Thomson devices, with emitted photon energies below $E_{p}=100 \mathrm{keV}$, the emission is generally provided by the collision between a high energy laser and a high brightness electron beam generated by linacs or storage rings [6], while more sophisticated schemes and interaction mechanisms are rapidly becoming a reality [13-17]. The most usual configuration is the head-to-head scattering, with the collision angle between the interaction beams $\alpha=180^{\circ}$, but also geometries with $\alpha=165^{\circ}$ or $\alpha=90^{\circ}$ have been tested [7]. The mean photon energy actually measured in the various sources ranges between 7 and

Published by the American Physical Society under the terms of the Creative Commons Attribution 3.0 License. Further distribution of this work must maintain attribution to the author(s) and the published article's title, journal citation, and DOI.
$70 \mathrm{keV}$, each device presenting a wide tunability. Experiments on the source characterization [18-21], on the transmission, dark field and phase contrast imaging, computed microtomography, K-edge techniques on phantom [22], biological [6,23], animal [24,25] and human [7] samples have been then successfully performed. The Thomson source SL_Thomson [26] at SPARC_LAB (INFN-LNF) [27] is based on the backscattering between the light pulse provided by the high energy Ti:sapphire laser FLAME [28] and the beam of the photoinjector SPARC [29]. The electron beam energy $E_{e}$ ranges between $30 \mathrm{MeV}$ and $180 \mathrm{MeV}$, producing Doppler blue shifted hard X-rays with energy $E_{p} \approx 4 E_{0} \gamma^{2}$ between 20 and $500 \mathrm{keV}$ $\left(E_{0}=1.55 \mathrm{eV}\right.$ is the photon energy of FLAME and $\gamma=60$ the electron Lorentz factor).

As regards the existing gamma Compton sources, the facility Higs [11], which represents the state of the art up to now, relies on the emission produced by the scattering of an electron beam and its FEL radiation. The total gamma-ray intensities (energy between 2 and $100 \mathrm{MeV}$ with linear and circular polarizations) can reach more than $10^{9}$ photons/ second with few percent energy resolution.

At the Nuclear Physics Pillar of the European laser facility ELI (Extreme Light Infrastructure)[30,31] an advanced Gamma System using a Yb:Yag collision laser is foreseen as a major component of the infrastructure, aiming at producing extreme gamma ray beams for nuclear physics and nuclear photonics. In this field, there are many 
strategic studies and applications for national security, nuclear waste treatment, nuclear medicine and fundamental studies in nuclear physics, dealing with the nucleus structure and the role of giant dipole resonances, with impact on astrophysics and star nucleosynthesis.

The polarization of the gamma rays is mandatory for measurements of the parity of the nuclear states, for the investigation of pygmy resonances and of the photodisintegration reactions. Measurements of the gamma polarization are usually done by analyzing the intensity spatial distribution detected on imaging plates [32]. Furthermore, the polarization of the Compton emission connected to the gamma-ray bursts and the active galactic nuclei permits us to distinguish between different emission mechanisms and to individuate the structures of the emission region.

The polarization properties of the Compton light are also usually exploited in Compton polarimeters, which are based on the asymmetry of the Compton cross section with respect to the spin of the electrons.

In this paper, a systematic study of the polarization of $\mathrm{X}$-gamma rays produced in the linear regime of the Thomson and Compton scattering is presented, in both classical and quantum schemes. After a brief review of the main characteristics of the Compton scattering, we compare the classical and the quantum approaches to the radiation polarization. Numerical results and analytical considerations let us establish the polarization level as a function of acceptance, bandwidth, and energy. Few sources have been considered: the first is the SPARC_LAB Thomson device, as an example of a $\mathrm{x}$-ray Thomson source, the second one, operating in the gamma range, is ELI-NP. Eventually, the typical parameters of a beam produced by a plasma accelerator has been used.

\section{GENERALITIES OF COMPTON SCATTERING}

The Thomson and Compton scattering is the collision between relativistic electrons and an electromagnetic field provided, for instance, by a high energy laser. The result of the process is the absorption of the colliding photons followed by emission of photons characterized by frequency upshift due to double relativistic Doppler effect. The geometry of the scattering is depicted in Fig. 1 [33]. The electron beam propagating along the $z$ direction impinges the laser radiation in the interaction point at an angle $\alpha$ close to $180^{\circ}$.

Each electron, characterized by normalized velocity $\underline{\beta}_{i}$, scatters photons with frequency:

$$
\nu_{p}=\nu_{0} \frac{1-\underline{e}_{k} \cdot \underline{\beta}_{i}}{1-\underline{n} \cdot \underline{\beta}_{i}+\frac{h \nu_{0}}{m c^{2} \gamma_{i}}\left(1-\underline{e}_{k} \cdot \underline{n}\right)}
$$

where $\nu_{0}$ is the frequency of the incident laser photon, $\underline{e}_{k}$ the unit vector of its direction, $\underline{n}$ is the direction of the scattered photon, $h$ the Planck constant, and $\gamma_{i}$ the electron

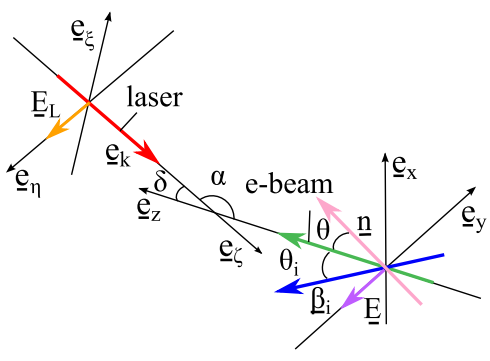

FIG. 1. Interaction geometry. $\left(\underline{e}_{x}, \underline{e}_{y}, \underline{e}_{z}\right)$ : laboratory frame. Laser: $\left(\underline{e}_{\xi}, \underline{e}_{\eta}, \underline{e}_{k}\right)$ : laser proper reference frame; red arrow: propagation direction, orange arrow: polarization versor. Electron beam: green arrow: average electron motion direction, blue arrow: ith electron motion direction, $\theta_{i}$ : angle of the ith electron. $\alpha$ : collision angle, $\delta=\pi-\alpha$. Radiation: pink arrow: observer direction, $\theta$ : observer angle, violet arrow: radiation polarization versor.

Lorentz factor. The last term in the denominator is related to the quantum red shift and becomes important when the Lorentz factor of the electron beam approaches the $\mathrm{GeV}$, when the desired bandwidth is very thin or when the laser energy is extremely high. Formula (1) describes the frequency-angle correlation. This feature is typical of sources of this kind, and allows to adjust the bandwidth. The distributions of the radiation total energy (a) and of the emitted photon number (b) as a function of the normalized photon frequency $\nu=\nu_{p} /\left(4 \nu_{0} \gamma^{2}\right)$ are shown in Fig. 2 .

\section{POLARIZATION IN THE CLASSICAL MODEL}

In the classical model [34], the electric field of the radiation in the far field approximation is given by:

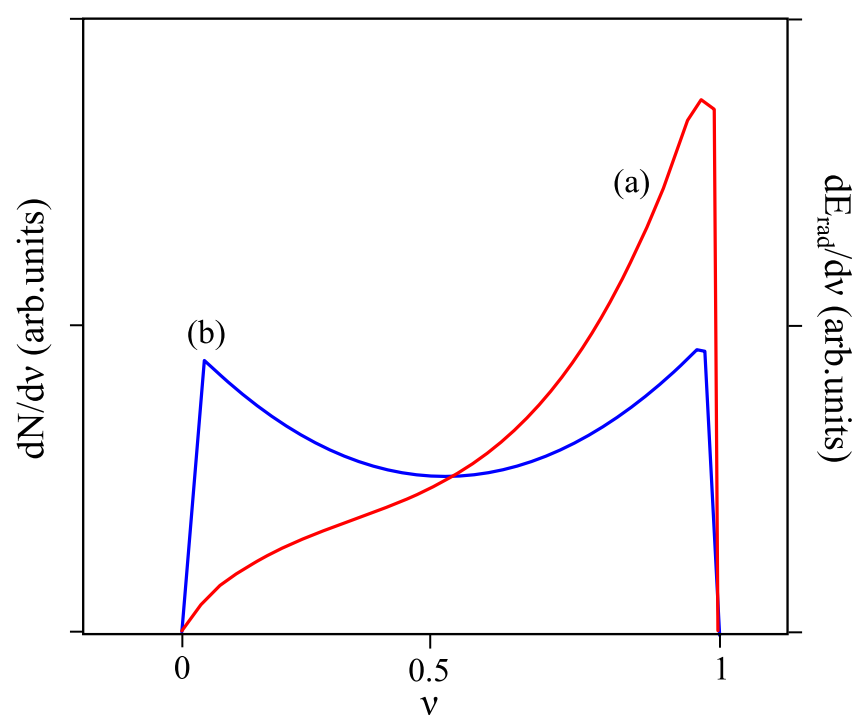

FIG. 2. Distributions of photon energy $E_{\text {rad }}$ (a) (right axis) and photon number $N_{\text {rad }}$ (left axis) (b) in linear scale, arbitary units, as function of the normalized photon frequency $\nu=\nu_{p} /\left(4 \nu_{0} \gamma^{2}\right)$. 


$$
\underline{E}=\frac{e}{c}\left[\frac{\underline{n} \times((n-\underline{\beta}) \times \stackrel{\beta}{\dot{\beta}})}{(1-\underline{\beta} \cdot \underline{n})^{3} R}\right]_{\mathrm{ret}}
$$

where $\underline{n}=(\sin \theta \cos \phi, \sin \theta \sin \phi, \cos \theta)$ is the direction of the observer at distance $R, \beta$ and $\dot{\beta}$ are, respectively, the velocity and the acceleration of the electron, and the expression is calculated at the retarded time.

The motion equation can be cast in the form:

$$
\dot{\beta}=-\frac{e}{m c \gamma}\left[\underline{E}_{L}\left(1-\underline{\beta} \cdot \underline{e}_{k}\right)+\underline{\beta} \cdot \underline{E}_{L}\left(\underline{e}_{k}-\underline{\beta}\right)\right] .
$$

If the laser propagation direction is along $z\left(\underline{e}_{k}=-\underline{e}_{z}\right)$ and its polarization is linear and directed along $y$, the acceleration is proportional to the vector $\underline{\lambda}=\underline{e}_{y}\left(1+\beta_{z}\right)+$ $\beta_{y}\left(-\underline{e}_{z}-\underline{\beta}\right)$.

Supposing, for the sake of simplicity, $\underline{\beta}=\beta_{z} \underline{e}_{z}$, in the linear limit (the velocity is considered not altered by the scattering) we obtain:

$$
\begin{aligned}
\underline{n} \times((\underline{n}-\underline{\beta}) \times \underline{\lambda})= & \left(1+\beta_{z}\right)\left[\underline{e}_{y}\left(-n_{x}^{2}-n_{z}\left(n_{z}-\beta_{z}\right)\right)\right. \\
& \left.+\underline{e}_{x} n_{x} n_{y}+\underline{e}_{z} n_{x}\left(n_{z}-\beta_{z}\right)\right] .
\end{aligned}
$$

The asymmetry of the radiation spot can therefore be deduced directly from (3), because:

$$
|E|^{2}(x, y=0)=\frac{4 e^{4} E_{L}^{2}}{m^{2} c^{4} \gamma^{2}\left(1-\beta_{z} \frac{z}{R}\right)^{6} R^{2}}\left(-\frac{1}{2 \gamma^{2}}+\frac{y^{2}}{R^{2}}\right)^{2}
$$

while:

$$
|E|^{2}(x=0, y)=\frac{e^{4} E_{L}^{2}}{m^{2} c^{4} \gamma^{6}\left(1-\beta_{z} \frac{z}{R}\right)^{6} R^{2}} .
$$

In Fig. 3 the profiles of the radiation along $\mathrm{x}$ and $\mathrm{y}$ are shown.

The relevant Stokes parameter $S_{3}$ is therefore:

$$
\begin{aligned}
S_{3} & =\frac{\left|E_{x}\right|^{2}-\left|E_{y}\right|^{2}}{|E|^{2}} \\
& =\frac{-\frac{1}{\gamma^{4}}-\frac{2}{\gamma^{2}} \sin ^{2} \theta \cos 2 \phi-\sin ^{4} \theta \cos 4 \phi}{\frac{1}{\gamma^{4}}+\frac{2}{\gamma^{2}} \sin ^{2} \theta \cos 2 \phi+\sin ^{4} \theta}
\end{aligned}
$$

and it vanishes at $z=R$ along the four curves:

$$
y= \pm x \pm \sqrt{2 x^{2}+\frac{R^{2}}{\gamma^{2}}} .
$$

Formula (6) can be analyzed in the limit $\theta \ll 1 / \gamma$ $(\gamma \sin \theta \ll 1)$ giving:

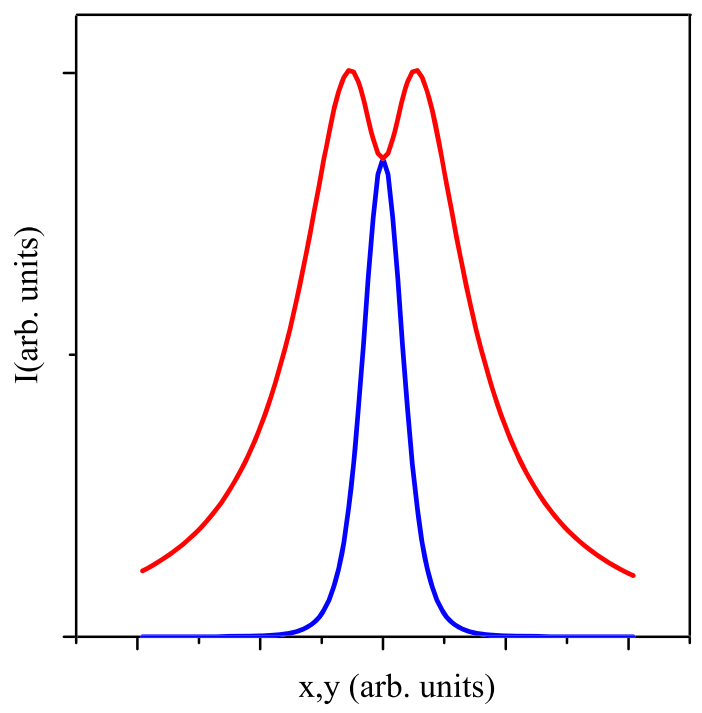

FIG. 3. Distribution of the radiation intensity along $\mathrm{x}$ (red) and y (blue).

$$
S_{3}=-\left[1-3 \gamma^{4} \sin ^{4} \theta(1-\cos 4 \phi)\right]
$$

while, if $\theta \geq 1 / \gamma(\gamma \sin \theta \gg 1)$ :

$$
S_{3}=-\left[\cos 4 \phi-+\frac{\cos 2 \phi}{\gamma^{2} \sin ^{2} \theta}(1-\cos 4 \phi)\right]
$$

showing the typical structure of a double cross. The central part of the pulse, within the $1 / \gamma$ circle, has almost the same polarization of the laser beam. At the border and outside this circle, the polarization changes, forming optical vortexes. Along the diagonals, polarization along $\mathrm{x}$ is dominant. Figure 4 represents the Stokes parameter for linear polarization as given by a classical Thomson code [35] on a screen, with electron beams with energies of $5 \mathrm{MeV}$, $53 \mathrm{MeV}, 530 \mathrm{MeV}$ and $2 \mathrm{GeV}$. The portion of the screen shown in the figures scales as $1 / \gamma$. With increasing $\gamma$, the polarization presents an almost complete similarity. Instead, the intensity, with increasing $\gamma$, develops a more pronounced dipolar structure and higher wings.

For a circular polarization, the Stokes parameter:

$$
S_{2}=\frac{\left|E_{r}\right|^{2}-\left|E_{l}\right|^{2}}{\left|E_{r}\right|^{2}+\left|E_{l}\right|^{2}}
$$

with $E_{r}$ and $E_{l}$ clockwise and counterclockwise components of the electric field, vanishes on the circle:

$$
\theta=\frac{1}{\gamma}
$$

and the profiles along $\xi=x, y$ are given by: 


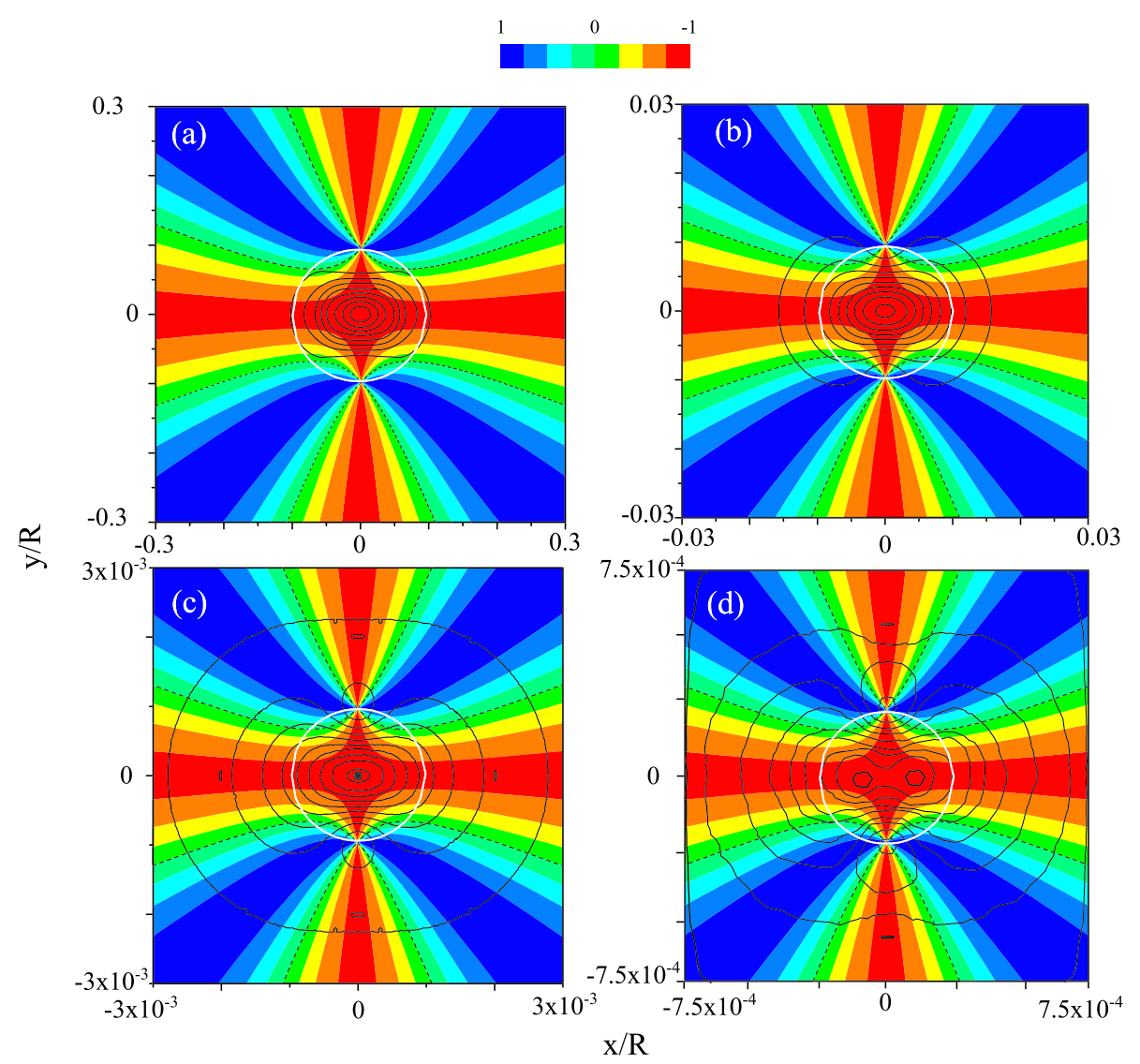

FIG. 4. Stokes parameter $S_{3}$ and level lines of radiation intensity in the plane $(x / R, y / R)$ (R being the distance between the source and the observation plane), (a) $E_{e}=5.3 \mathrm{MeV}$, (b) $E_{e}=53 \mathrm{MeV}$ (c) $E_{e}=530 \mathrm{MeV}$ (d) $E_{e}=2000 \mathrm{MeV}$. Classical treatment. In white, the circle $1 / \gamma$. The dashed curves are the solution of Eq. (7).

$$
\left|E_{r}\right|^{2}=\frac{2 e E_{L}^{2}}{m^{2} c^{4} \gamma^{2}\left(1-\beta_{z} \frac{z}{R}\right)^{6}} \frac{\xi^{4}}{R^{6}}
$$

In the linear approximation, the radiation intensity (4)-(5) and (11) are proportional to the laser intensity $\left|E_{L}\right|^{2}$, while the Stokes parameters (6) and (10) turn out to be independent of it.

The addition of an emittance to the electron beam can be analyzed by supposing that the velocity of the ith electron exhibits all components $\underline{\beta}=\beta_{x} \underline{e}_{x}+\beta_{y} \underline{e}_{y}+\beta_{z} \underline{e}_{z}$ with $\beta_{x, y} \ll 1$.

The components of the electric field can be written as:

$$
\begin{aligned}
& E_{x} \approx\left(n_{x}-\beta_{x}\right)\left[2 n_{y}-\beta_{y}\right] \\
& E_{y} \approx-n_{y} \beta_{y}-\frac{1}{\gamma^{2}}-\beta_{x}^{2}+n_{x}^{2}-n_{y}^{2}+2 n_{x} \beta_{x} .
\end{aligned}
$$

Summing over all electrons, we can express the components of the electric field and hence the Stokes parameters as a function of the emittance $\epsilon_{x, y} \approx \Delta \beta_{x, y} \sigma_{x, y}$.

Misalignment and angular displacements of the photons due to jitters or alignment error between the source and the collimator produce a further degradation of the polarization. Angular displacements can be treated in a way similar to the emittance, introducing in the electric field the components of the electron velocity: $\beta_{x}=\beta \sin \theta_{0} \cos \phi_{0}$, $\beta_{y}=\beta \sin \theta_{0} \sin \phi_{0}, \beta_{z}=\beta \cos \theta_{0}, \theta_{0}$ and $\phi_{0}$ being the angles between the axis of the electron beam and the axis of the collimator. The following expression, obtained by summing over all electrons, gives the dependence of the average value of the modulus of the Stokes parameter on the angular jitters as a function of $\theta_{0}$ and $\phi_{0}$ :

$$
\begin{gathered}
\left|\left\langle S_{3}\right\rangle\right|=1-\frac{51}{4} \gamma^{4} \sin ^{4} \theta_{0} \sin 2 \phi_{0} \\
\text { IV. POLARIZATION IN THE } \\
\text { QUANTUM TREATMENT }
\end{gathered}
$$

\section{POLARIZATION IN THE QUANTUM TREATMENT}

An analogous study can be done within the quantum model. The cross section in the electron rest frame can be written as:

$$
\frac{d \sigma}{d \Omega}=\frac{r_{0}^{2}}{4}\left(\frac{\nu_{p}}{\nu_{0}}\right)^{2}\left[\frac{\nu_{p}}{\nu_{0}}+\frac{\nu_{0}}{\nu_{p}}-2+4\left(\underline{e}_{0} \cdot \underline{e}_{p}\right)^{2}\right] .
$$

In the laboratory frame, instead, we have:

$$
\frac{d \sigma^{\prime}}{d \Omega}=\frac{r_{0}^{2}}{4}\left(\frac{\nu_{p}^{\prime}}{\nu_{0}^{\prime}}\right)^{2}\left[\frac{\nu_{p}^{\prime}}{\nu_{0}^{\prime}}+\frac{\nu_{0}^{\prime}}{\nu_{p}^{\prime}}-2+4\left(\underline{e}_{0}^{\prime} \cdot \underline{e}_{p}^{\prime}\right)^{2}\right]
$$


TABLE I. Electron beam, laser, and collision specifics. $E_{e}$ : electron beam energy, $\lambda_{L}$ : laser wavelength, $E_{L}$ : total laser energy, $\delta$ : complementary collision angle, $w_{0}$ : laser waist, t laser time duration, $a_{0}$ : laser parameter.

\begin{tabular}{|c|c|c|c|c|c|c|c|c|c|c|}
\hline Beam & $E_{e}$ & Charge & Emittance & Energy spread & $\lambda_{L}$ & $E_{L}$ & $\delta$ & $w_{0}$ & $\tau$ & $a_{0}$ \\
\hline 1, SPARC & $30 \mathrm{MeV}$ & $0.25 \mathrm{nC}$ & $1 \mathrm{~mm} \mathrm{mrad}$ & $5 \times 10^{-4}$ & $800 \mathrm{~nm}$ & $1 \mathrm{~J}$ & $0^{\circ}$ & $28 \mu \mathrm{m}$ & $1.5 \mathrm{ps}$ & 0.32 \\
\hline 2, ELI-NP & $234 \mathrm{MeV}$ & $0.25 \mathrm{nC}$ & $0.5 \mathrm{~mm} \mathrm{mrad}$ & $7 \times 10^{-4}$ & $520 \mathrm{~nm}$ & $0.2 \mathrm{~J}$ & $8^{\circ}$ & $28 \mu \mathrm{m}$ & $1.5 \mathrm{ps}$ & 0.064 \\
\hline 3, ELI-NP & $529 \mathrm{MeV}$ & $0.25 \mathrm{nC}$ & $0.5 \mathrm{~mm} \mathrm{mrad}$ & $7 \times 10^{-4}$ & $520 \mathrm{~nm}$ & $0.4 \mathrm{~J}$ & $8^{\circ}$ & $28 \mu \mathrm{m}$ & $1.5 \mathrm{ps}$ & 0.064 \\
\hline
\end{tabular}

and:

$$
\left[\frac{d \sigma}{d \Omega}\right]^{\prime}=\frac{r_{0}^{2}}{4 \gamma^{2}\left(1-\underline{\beta} \cdot \underline{e}_{k}\right)^{2}}\left(\frac{\nu_{p}}{\nu_{0}}\right)^{2} X
$$

$X=\frac{\nu_{0}}{\nu_{p}} \frac{1-\underline{\beta} \cdot \underline{e_{k}}}{1-\underline{\beta} \cdot \underline{n}}+\frac{\nu_{p}}{\nu_{0}} \frac{1-\underline{\beta} \cdot \underline{n}}{1-\underline{\beta} \cdot \underline{e}_{k}}-2+4\left(\underline{e}_{0}^{\prime} \cdot \underline{e}_{p}^{\prime}\right)^{2}$

\section{NUMERICAL RESULTS}

Three typical examples are presented. First the case of the Thomson source SPARC_LAB with parameters similar to those used in the first stage of the experiment [36], then the case of the gamma source ELI-NP. The third case is a source based on a typical electron beam obtained by plasma acceleration.

\section{A. Thomson Source SPARC_LAB}

The polarization of a Thomson source have been studied in the linear classical framework [35]. Typical parameters of the electron beam and laser are summarized in Table I (line 1) and recall the specifics of the SPARC_LAB source in the first phase of operation. The laser parameter
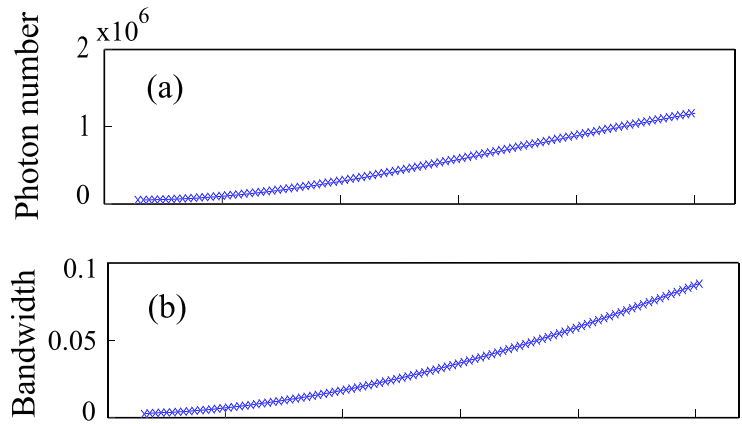

Supposing that, as before, $\underline{\beta}=\beta_{z} \underline{e}_{z}, \underline{e}_{0}=\underline{e}_{y}, \underline{e}_{k}=-\underline{e}_{z}$, and that the last three terms in the denominator are negligible, the direct calculation of the Stokes parameter lead to:

$$
S_{3}=\frac{-\frac{1}{\gamma^{4}}-\frac{2}{\gamma^{2}} \sin ^{2} \theta \cos 2 \phi-\sin ^{4} \theta \cos 4 \phi}{\frac{1}{\gamma^{4}}+\frac{2}{\gamma^{2}} \sin ^{2} \theta \cos 2 \phi+\sin ^{4} \theta}
$$

expression analogous to Eq. (6).

The average value of the Stokes parameter over the collection area for $\gamma \theta \lesssim 1$ is given by:

$$
\left|\left\langle S_{3}\right\rangle\right| \approx 1-\frac{3}{4} \gamma^{4} \theta_{\max }^{4}
$$

By expressing the radiation polarization versor $\underline{e}_{p}$ $\cos \phi \underline{e}_{y}$, and $\underline{e}_{p 2}=\cos \phi \cos \theta \underline{e}_{x}+\sin \phi \cos \theta \underline{e}_{y}-\sin \theta \underline{e}_{z}$, $S_{3}=\frac{\left(\underline{e}_{0}^{\prime} \cdot \underline{e}_{p}^{\prime}\right)_{\underline{e}_{p}=\underline{e}_{p 1}}^{2}-\left(\underline{e}_{0}^{\prime} \cdot \underline{e}_{p}^{\prime}\right)_{\underline{e}_{p}=\underline{e}_{p 2}}^{2}}{\left(\underline{e}_{0}^{\prime} \cdot \underline{e}_{p}^{\prime}\right)_{\underline{e}_{p}=\underline{e}_{p 1}}^{2}+\left(\underline{e}_{0}^{\prime} \cdot \underline{e}_{p}^{\prime}\right)_{\underline{e}_{p}=\underline{e}_{p 2}}^{2}+\frac{\nu_{0}}{2 \nu_{p}} \frac{1-\underline{\beta} \underline{e}_{k}}{1-\underline{\beta} \cdot \underline{\underline{n}}}+\frac{\nu_{p}}{2 \nu_{0}} \frac{1-\bar{\beta} \cdot \underline{\underline{e}}}{1-\underline{\underline{e}}-\underline{\underline{e}}}-1}$

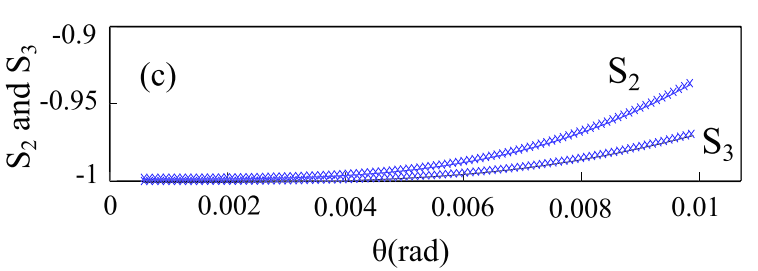

FIG. 5. SPARC case (beam 1): (a) Total number of emitted photons, (b) relative bandwidth and (c) average Stokes parameter with a linearly $\left(S_{3}\right)$ and circularly $\left(S_{2}\right)$ polarized laser as functions of the acceptance angle $\theta$ with parameters similar to the SPARC one in the first phase of the experimental operation. Simulation done with CAIN. 

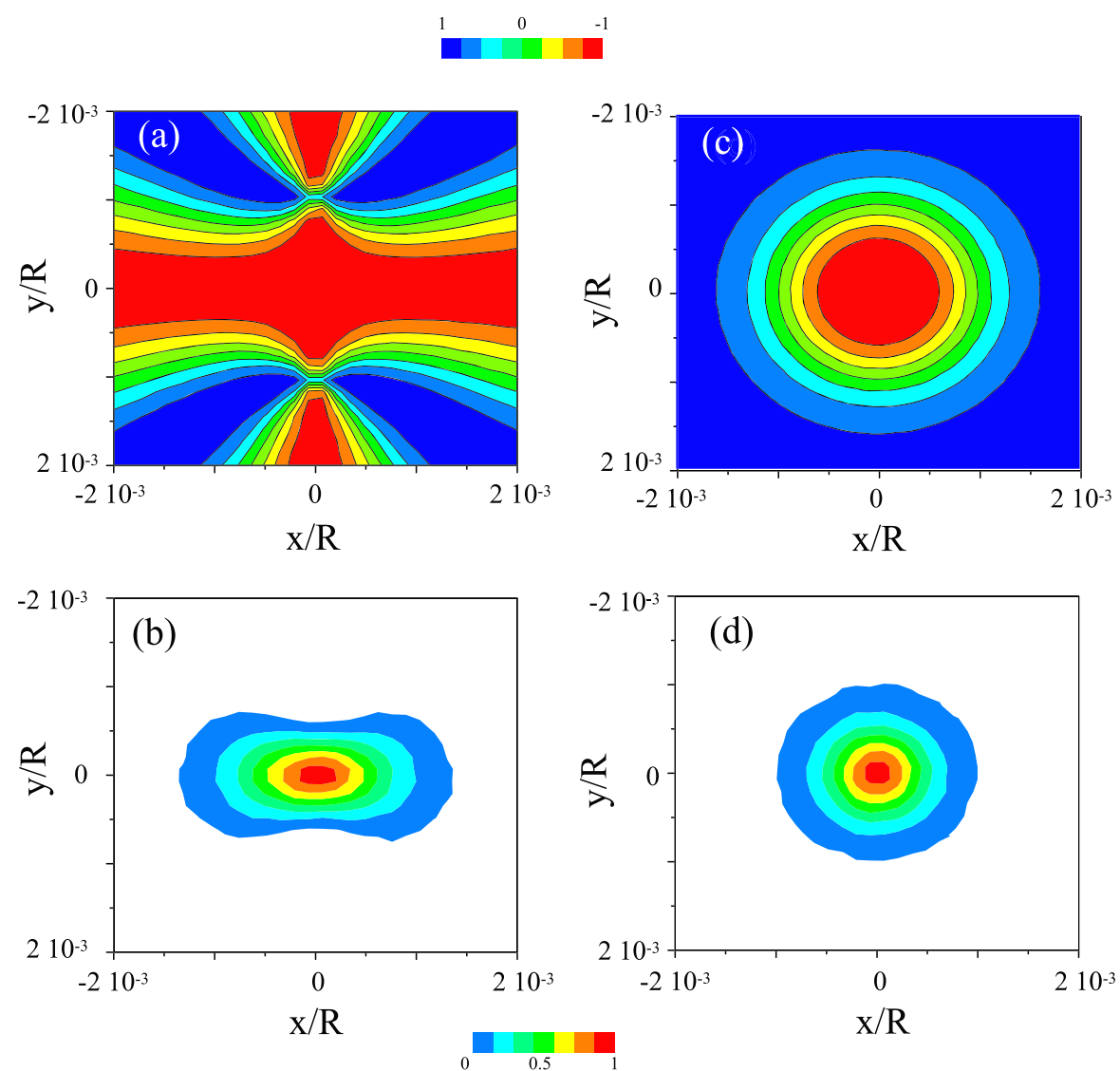

FIG. 6. ELI-NP case (beam 2): Stokes parameter $S_{3}$ (a) and $S_{2}$ (c) and photon distribution (b) and (d) for linear (left) and circular (right) polarization, with photon energy $10 \mathrm{MeV}$.
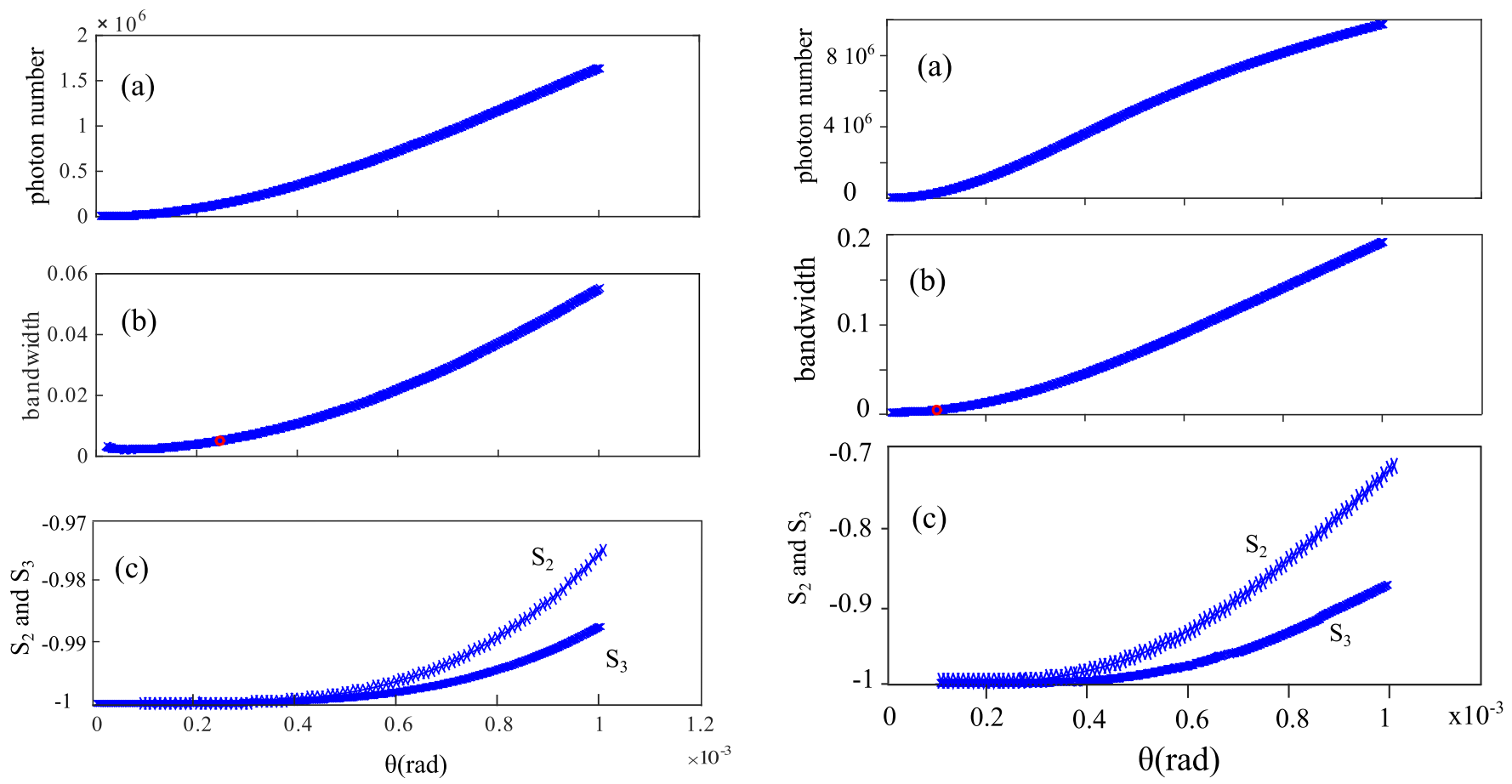

FIG. 7. ELI-NP case (beam 2): (a) photon number, (b) relative bandwidth, and (c) Stokes parameters $S_{2}$ and $S_{3}$ for gamma rays at $2 \mathrm{MeV}$, linear and circular laser polarizations.

FIG. 8. ELI-NP case (beam3) (a) Photon number, (b) relative bandwidth, and (c) Stokes parameters for gamma rays at $10 \mathrm{MeV}$ (beam 3), linear and circular laser polarizations. 
TABLE II. Average values of flux and of the Stokes parameters $\left\langle S_{3}\right\rangle$ and $\left\langle S_{2}\right\rangle$ for both linear and circular laser polarization and various working points, in the bandwidth $b w_{1}=5 \times 10^{-3}$ and for a larger acceptance, corresponding to a bandwidth of $b w_{2}=0.2$. Laser energy at $0.2 \mathrm{~J}$. In the cases with star, the laser energy is $0.4 \mathrm{~J}$.

\begin{tabular}{lccccccc}
\hline \hline$E_{e}(\mathrm{MeV})$ & $E_{p}(\mathrm{MeV})$ & Flux $\left(b w_{1}\right)$ & $\left\langle S_{3}\right\rangle b w_{1}$ & $\left\langle S_{2}\right\rangle b w_{1}$ & Flux $\left(b w_{2}\right)$ & $\left\langle S_{3}\right\rangle b w_{2}$ & $\left\langle S_{2}\right\rangle b w_{2}$ \\
\hline 165 & 1 & $1.48 \times 10^{5}$ & $>0.999$ & $>0.999$ & $4 \times 10^{6}$ & 0.85 & 0.65 \\
234 & 2 & $1.35 \times 10^{5}$ & $>0.999$ & $>0.999$ & $4 \times 10^{6}$ & 0.85 & 0.68 \\
311 & 3.5 & $1.09 \times 10^{5}$ & $>0.999$ & $>0.999$ & $3.9 \times 10^{6}$ & 0.85 & 0.68 \\
$* 529$ & 10 & $2.97 \times 10^{5}$ & $>0.999$ & $>0.999$ & $10^{7}$ & 0.86 & 0.72 \\
$* 605$ & 13 & $2.67 \times 10^{5}$ & $>0.999$ & $>0.999$ & $9.5 \times 10^{6}$ & 0.86 & 0.71 \\
$* 750$ & 19.5 & $2.37 \times 10^{5}$ & $>0.999$ & $>0.999$ & $10^{7}$ & 0.87 & 0.7 \\
\hline \hline
\end{tabular}

$a_{0}=0.42 \times 10^{16} \sqrt{\frac{E_{L}(J)}{\tau(p s)}} \frac{1}{w_{0}(\mu m) \nu_{0}}$ is about 0.3 for the parameters chosen, allowing the use of a linear model.

The electron beam has been simulated by T-STEP [37] and ASTRA [38], with a charge of $250 \mathrm{pC}$ and a final focus of about $20 \mu \mathrm{m} \mathrm{rms}$. The collision results in a photon yield larger than $10^{6}$ in a bandwidth of about $5 \%$. A strong level of polarization in the bandwidth has been obtained, as shown in Fig. 5, where the total number of emitted photons (a), the relative bandwidth (b) and the average Stokes parameter with a linearly and circularly polarized laser (c) are shown as functions of the acceptance angle. The variation of the acceptance angle simulates the presence along the radiation beam line of a collimator that selects only part of the radiation and permits us to control the bandwidth.

\section{B. Compton source ELI-NP}

The polarization of the ELI-NP source [31] has been studied with CAIN [39], which is a Monte Carlo code based on the Volkov solution of the Dirac equation. The reference electron beams for various working points and the nominal laser parameters and interaction conditions [30] have been used (see Table I). In particular, in our calculations, the laser is considered $100 \%$ polarized [40]. Since, however, the expected laser polarization at ELI-NP is higher than $98 \%$, the effective polarization figures reported in graphs and tables should be normalized by this factor. The polarization degree is connected to the momentum distribution of the photons, so the polarization shape appears only after propagation of the photons up to the far zone. Figure 6 gives the distribution on the screen of the Stokes parameter and of the photon intensity in the far zone, with photons at $10 \mathrm{MeV}$ for linear and circular polarization, showing a pattern very similar to the classical case.

Figures 7 and 8 give the photon number, the relative bandwidth, and the average values of $S_{3}$ and $S_{2}$ as a function of the acceptance angle $\theta$ for the working points at $2 \mathrm{MeV}$ and $10 \mathrm{MeV}$. The average values of flux and of the Stokes parameters $\left\langle S_{3}\right\rangle$ and $\left\langle S_{2}\right\rangle$ for both linear and circular
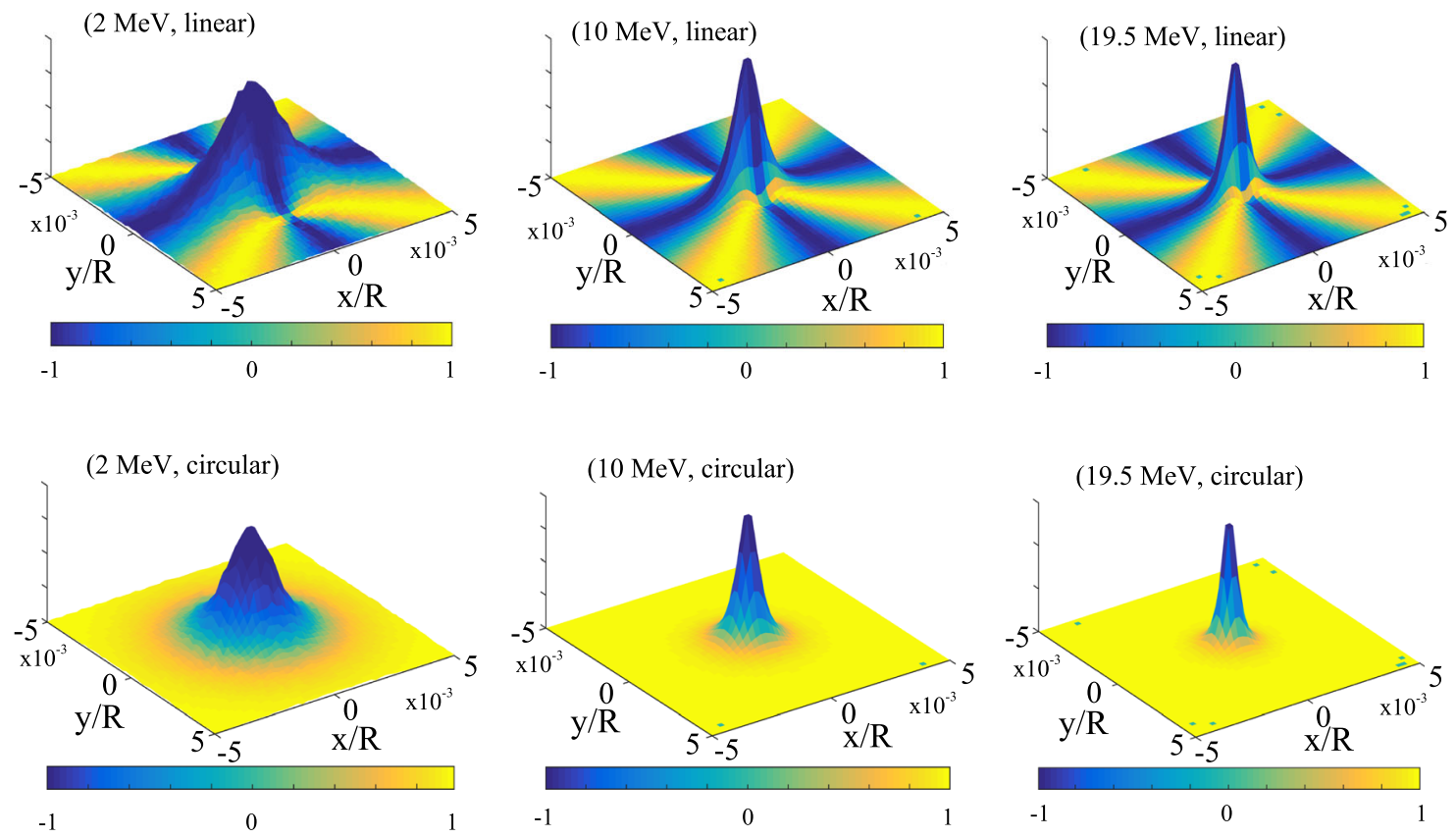

FIG. 9. ELI-NP case: intensity-polarization graphs for photons at 2,10,19.5 MeV, with linear and circular laser polarization. 

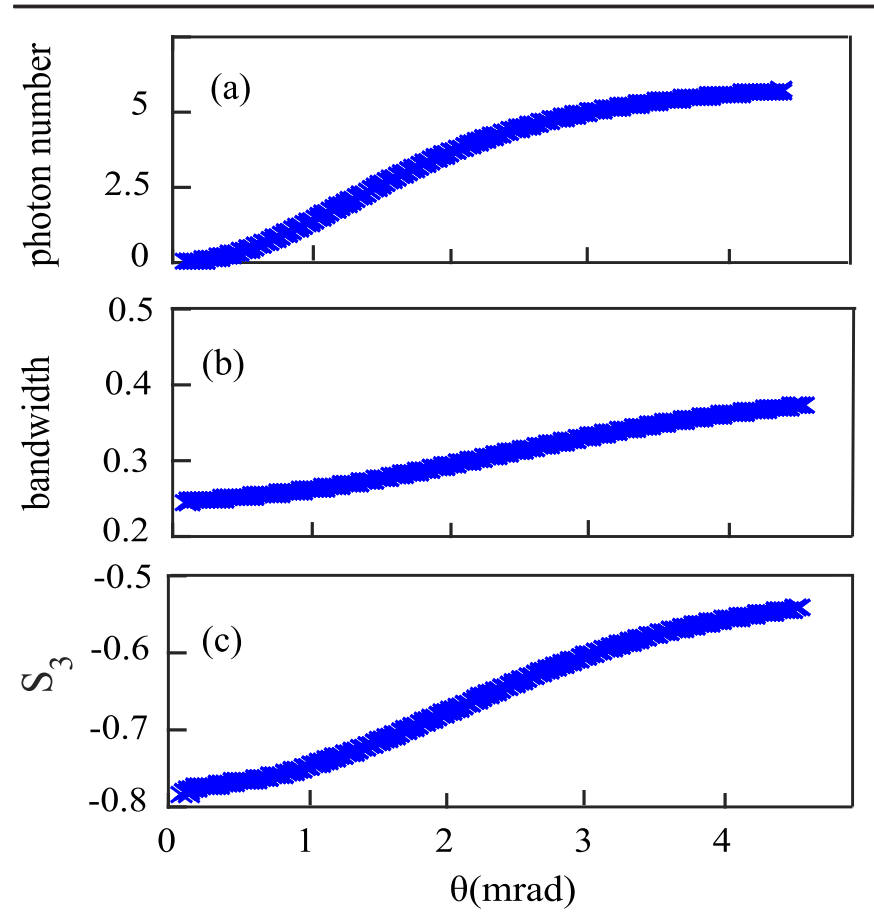

FIG. 10. (a) Photon flux, (b) bandwidth, and (c) Stokes parameter $S_{3}$ vs acceptance angle for a typical plasma beam.

laser polarization and various working points, calculated in the typical ELI bandwidth $b w_{1}=5 \times 10^{-3}$ and for a larger acceptance, corresponding to a bandwidth of $b w_{2}=0.2$ are listed in Table II.

The results show that, while for the cases at bandwidth $b w_{1}=5 \times 10^{-3}$ the polarization is always close to 1 [see Eq. (9)], the depolarization increases with the bandwidth, reaching $15 \%$ at $b w_{2}=0.2$ in the case of linear polarization and also more than $30 \%$ for circular polarization, a situation which appears to be quite critical for users requiring tight control of the polarization of the source.

In Fig. 9, a synthetic view of both intensity and polarization is shown for several ELI-NP working points.

\section{Typical beam produced by a plasma accelerator}

Plasma accelerated electron beams have been recently generated and measured [13]. Typical value of the

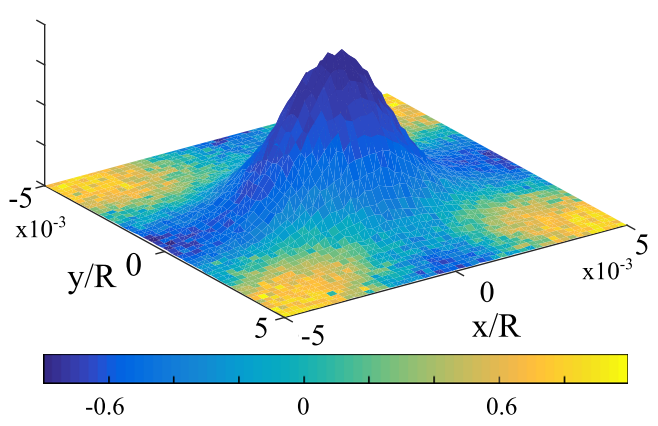

FIG. 11. Intensity-polarization graph for the plasma acceleration source. parameters are $120 \mathrm{pC}$ of charge and a transverse rms dimension of about $1.5 \mu \mathrm{m}$. The interaction with a laser field has produced about $10^{8}$ photons with a spectrum extending up to $100 \mathrm{MeV}$ with a divergence of $18 \mathrm{mrad}$. We have assumed a beam of this kind, with emittance of $1 \mathrm{~mm}$ mrad and an energy spread of $1 \%$, interacting with a laser similar to that of the previous ELI-NP case.

In Fig. 10 the photon flux, bandwidth, and Stokes parameter are reported, while Fig. 11 presents the intensitypolarization graph of the radiation. The strong focusing of the electron beam leads to a considerably high flux. However, the not negligible value of the emittance, connected to the presence of large transverse momenta, makes the bandwidth always larger than about 30\%. Despite the small energy spread, the large value of the minimum bandwidth is set by the angular spread of the electron beam. As a consequence of the emittance, also the polarization deteriorates. The modulus of the Stokes parameter, in fact, is never higher than $80 \%$. These estimates lead to the considerations that an all optical gamma source based on a plasma accelerated electrons cannot guarantee the narrow relative bandwidth $(\sim 0.5 \%)$ and the high polarization $(\gtrsim 98 \%)$ conditions required in nuclear photonics experiments.

\section{CONCLUSIONS}

We have studied the polarization variation in the two typical situations, the $\mathrm{X}$ rays Thomson source at SPARC_LAB and the gamma source ELI-NP. In the first case, with bandwidths up to $10 \%$, a contained reduction $(<10 \%)$ in the average polarization occurs. In the last case, for the nominal ELI-NP bandwidth $5 \times 10^{-3}$, the polarization is always close to 1 . For applications requiring larger bandwidth, however, a degradation of the polarization up to $30 \%$ must be taken into account. In addition, an all optical gamma source based on a plasma accelerated electron beam cannot guarantee narrow bandwidth and high polarization operational conditions required in nuclear photonics experiments.

[1] C. M. Guenther, B. Pfau, R. Mitzner, B. Siemer et al., Nat. Photonics 5, 99 (2011).

[2] F. Tavella, N. Stojanovic, G. Geloni, and M. Gensch, Nat. Photonics 5, 162 (2011).

[3] I. V. Pogorelsky, I. Ben-Zvi, T. Hirose, S. Kashiwagi et al., Phys. Rev. ST Accel. Beams 3, 090702 (2000).

[4] W. Brown, S. Anderson, C. Barty, S. Betts, R. Booth, J. Crane et al., Phys. Rev. ST Accel. Beams 7, 060702 (2004); F. V. Hartemann, A. M. Tremaine, S. G. Anderson, C. P. J. Barty, S. M. Betts, R. Booth, W. J. Brown, J. K. Crane, R. R. Cross, D. J. Gibson, D. N. Fittinghoff, J. Kuba, G. P. Le Sage, D. R. Slaughter, A. J. Wootton, E. P. Hartouni, P. T. Springer, J. B. Rosenzweig, and A. K. Kerman, Laser Part. Beams 22, 221 (2004). 
[5] M. Babzien et al., Phys. Rev. Lett. 96, 054802 (2006).

[6] M. Bech, O. Bunk, C. David, R. Ruth, J. Rifkin, R. Loewen, R. Feidenhans'l, and F. Pfeiffer, J. Synchrotron Radiat. 16, 43 (2009).

[7] R. Kuroda, H. Toyokawa, M. Yasumoto, H. IkeuraSekiguchi, M. Koike, K. Yamada, T. Yanagida, T. Nakajyo, F. Sakai, and K. Mori, Nucl. Instrum. Methods Phys. Res., Sect. A 637, S183 (2011).

[8] Y. Du, L. Yan, J. Hua, Q. Du, Z. Zhang, R. Li, H. Qian, W. Huang, H. Chen, and C. Tang, Rev. Sci. Instrum. 84, 053301 (2013).

[9] A. Jochmann, A. Irman, U. Lehnert, J. P. Couperus et al., Nucl. Instrum. Methods Phys. Res., Sect. B 309, 214 (2013).

[10] G. Priebe et al., Laser Part. Beams 26, 649 (2008); D. Laundy, G. Priebe, S. P. Jamison, D. M. Graham, P. J. Phillips, S. L. Smith, Y. Saveliev, S. Vassilev, and E. A. Seddon, Nucl. Instrum. Methods Phys. Res., Sect. A 689, 108 (2012).

[11] C. Sun and Y. K. Wu, Phys. Rev. ST Accel. Beams 14, 044701 (2011).

[12] D. J. Gibson, F. Albert, S. G. Anderson, S. M. Betts et al., Phys. Rev. ST Accel. Beams 13, 070703 (2010).

[13] K. T. Phuoc, S. Corde, C. Thaury, V. Malka, A. Tafzi, J. P. Goddet, R. C. Shah, S. Sebban, and A. Rousse, Nat. Photonics 6, 308 (2012).

[14] V. Petrillo, L. Serafini, and P. Tomassini, Phys. Rev. ST Accel. Beams 11, 070703 (2008).

[15] N. D. Powers, I. Ghebregziabher, G. Golovin, C. Liu, S. Chen, S. Banerjee, J. Zhang, and D. P. Umstadter, Nat. Photonics 8, 28 (2014).

[16] V. Petrillo, A. Bacci, C. Curatolo et al., Phys. Rev. ST Accel. Beams 17, 020706 (2014).

[17] A. Debus, M. Bussmann, M. Siebold, A. Jochmann, U. Schramm, T. E. Cowan, and R. Sauerbrey, Appl. Phys. B 100, 61 (2010).

[18] A. Jochmann, A. Irman, M. Bussmann, J. P. Couperus, T. E. Cowan, A. D. Debus, M. Kuntzsch, K. W. D. Ledingham, U. Lehnert, R. Sauerbrey, H. P. Schlenvoigt, D. Seipt, Th. Stohlker, D. B. Thorn, S. Trotsenko, A. Wagner, and U. Schramm, Phys. Rev. Lett. 111, 114803 (2013).

[19] H. Ikeura-Sekiguchi, R. Kuroda, M. Yasumoto, H. Toyokawa, M. Koike, K. Yamada, F. Sakai, K. Mori, K. Maruyama, H. Oka, and T. Kimata, Appl. Phys. Lett. 92, 131107 (2008).

[20] M. Babzien, I. Ben-Zvi, K. Kusche, I. V. Pavlishin, I. V. Pogorelsky, D. P. Siddons, V. Yakimenko, D. Cline, F. Zhou, T. Hi-rose, Y. Kamiya, T. Kumita, T. Omori,
J. Urakawa, and Kaoru Yokoya, Phys. Rev. Lett. 96, 054802 (2006).

[21] Y. Sakai et al., Phys. Rev. ST Accel. Beams 18, 060702 (2015).

[22] K. Achterhold, M. Bech, S. Schleede, G. Potdevin, R. Ruth, R. Loewen, and F. Pfeiffer, Sci. Rep. 3, 1313 (2013); S. Schleede, M. Bech, K. Achterhold, G. Potdevin, M. Gifford, R. Loewen, C. Limborg, R. Ruth, and F. Pfeiffer, J. Synchrotron Radiat. 19, 525 (2012).

[23] B. Golosio, M. Endrizzi, P. Oliva, P. Delogu, M. Carpinelli, I. Pogorelsky, and V. Yakimenko, Appl. Phys. Lett. 100, 164104 (2012).

[24] F. G. Meinel, F. Schwab, S. Schleede, M. Bech, J. Herzen, K. Achterhold, S. Auweter, F. Bamberg, A. O. Yildirim, A. Bohla, O. Eickelberg, R. Loewen, M. Gifford, R. Ruth, M. F. Reiser, F. Pfeiffer, and K. Nikolaou, PLoS One 8, e59526 (2013); S. Schleede, F. G. Meinel, M. Bech et al., Proc. Natl. Acad. Sci. U.S.A. 109, 17880 (2012).

[25] F. Schwab, S. Schleede, D. Hahn et al., Z. Med. Phys. 23, 236 (2013).

[26] P. Oliva, A. Bacci, U. Bottigli, M. Carpinelli et al., Nucl. Instrum. Methods Phys. Res., Sect. A 615, 93 (2010).

[27] M. Ferrario, D. Alesini, M. P. Anania, A. Bacci et al., Nucl. Instrum. Methods Phys. Res., Sect. B 309, 183 (2013).

[28] L. A. Gizzi, A. Bacci, S. Betti et al., Eur. Phys. J. Spec. Top. 175, 3 (2009).

[29] L. Giannessi et al., Phys. Rev. ST Accel. Beams 14, 060712 (2011).

[30] O. Adriani et al., arXiv:1407.3669.

[31] A. Bacci et al., J. Appl. Phys. 113, 194508 (2013).

[32] S. Miyamoto, Y. Asano, S. Amano, D. Li, K. Imasaki, H. Kinugasa, Y. Shoji, T. Takagi, and T. Mochizuki, Radiation Measurements 41, S179 (2006).

[33] V. Petrillo et al., Nucl. Instrum. Methods Phys. Res., Sect. A 693, 1095 (2012).

[34] J. D. Jackson, Classical Electrodynamics, 3rd ed. (Wiley, New York, 1998).

[35] P. Tomassini et al., IEEE Trans. Plasma Sci. 36, 1782 (2008).

[36] C. Vaccarezza et al. (private communication).

[37] T-step is an upgraded version of Parmela, see: L. M. Young, PARMELA, Los Alamos National Laboratory Report No. LA-UR-96-1835.

[38] Floetmann, Astra, /http://desy.de/mpyo/Astra_ dokumentation/.

[39] K. Yokoya, /http://www-acc-theory.kek.jp/members/cainS/ (1985).

[40] K. Dupraz et al., Phys. Rev. ST Accel. Beams 17, 033501 (2014). 